\title{
Effect of a Modified Irrigation Protocol on the Cleanliness of Moderately Curved Canals
}

\author{
Garance Voegeli ${ }^{1} \quad$ Enrico Di Bella ${ }^{2} \quad$ Mustapha Mekki $^{3} \quad$ Pierre Machtou ${ }^{4} \quad$ Serge Bouillaguet ${ }^{1}$ \\ ${ }^{1}$ University Clinics of Dental Medicine, Faculty of Medicine, \\ University of Geneva, Geneva, Switzerland \\ ${ }^{2}$ Department of Economics and Business Studies, Faculty of \\ Economics, University of Genoa, Genoa, Italy \\ ${ }^{3}$ Biomaterials Unit, Section of Dental Medicine, Faculty of Medicine, \\ University of Geneva, Geneva, Switzerland \\ ${ }^{4}$ Faculty of Odontology, Paris, France \begin{abstract}
Switzerland (e-mail: garance.voegeli@unige.ch).
Serge Bouillaguet, University Clinics of Dental Medicine, Faculty of Medicine, University of Geneva, Geneva, Switzerland (e-mail: serge.bouillaguet@unige.ch).
\end{abstract} \\ Address for correspondence Garance Voegeli, University Clinics of \\ Dental Medicine, Faculty of Medicine, University of Geneva, Geneva,
}

Eur J Dent:2021;5:90-95

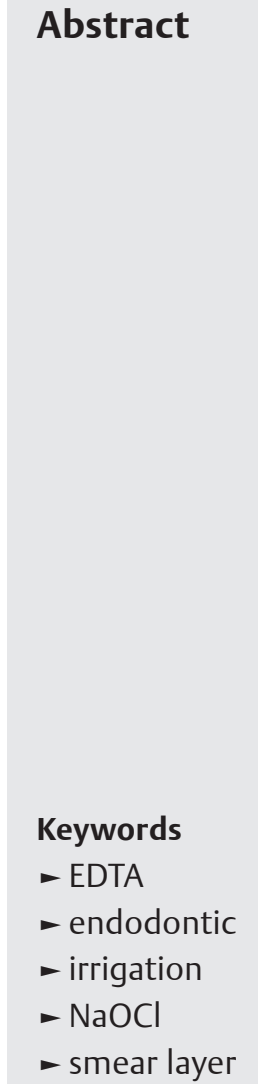

Objectives This study tested the hypothesis that modifying the sequence of sodium hypochlorite $(\mathrm{NaOCl})$ /ethylene diamine tetra-acetic acid (EDTA) irrigation during root canal shaping would improve apical cleanliness in moderately curved canals.

Materials and Methods Forty-five root canals were prepared using Protaper Gold with three irrigation protocols. Standard irrigation (SI) used $0.5 \mathrm{~mL} 3 \% \mathrm{NaOCl}$ between each instrument, followed by $5 \mathrm{~mL}$ 17\% EDTA manually agitated for $30 \mathrm{sec}$ onds. Reverse irrigation (RI) used $0.5 \mathrm{~mL}$ of $17 \%$ EDTA between each instrument, then $5 \mathrm{~mL}$ of $3 \% \mathrm{NaOCl}$, manually agitated for 30 seconds. Reverse irrigation plus (RI+) was similar to $\mathrm{RI}$, except $\mathrm{NaOCl}(5 \mathrm{~mL})$, used as a final rinse, was allowed to interact for 3 minutes with dentin before manual agitation (30 seconds).

Root canal cleanliness was evaluated under the scanning electron microscope (SEM) (Hulsmann score); the chemical composition of dentin after irrigation was analyzed by energy dispersive X-ray (EDX).

Statistical Analysis Results were compared using Kruskal-Wallis ANOVA by ranks and Wilcoxon matched paired posthoc tests. A Chi-square test assessed whether the best cleanliness would demonstrate a significant association with one irrigation protocol; odds ratio calculation was performed using score " 1 " versus score " 2 or more" $(2+)(p<0.05)$.

Results In the apical region, cleanliness was better in $\mathrm{RI}+$ than $\mathrm{SI}$ and both significantly better than RI. Odd ratios indicate that the cleanliness in $\mathrm{RI}+$ was significantly better than $\mathrm{RI}$ and $\mathrm{SI}$ groups ( $p<0.000$ and 0.003 , respectively). Independently of the irrigation protocol, EDX analyses showed no chemical alteration of root dentin.

Conclusions Using 17\% EDTA during shaping, followed by $3 \% \mathrm{NaOCl}$ rinse for 3 minutes, improved apical cleanliness without inducing erosion of dentin.

\section{Introduction}

The success of endodontic therapy strongly depends on proper shaping and cleaning of the root canal system before three-dimensional (3D) filling. Whereas shaping is primarily aimed at cutting infected dentin and enlarging root canals, cleaning mainly consists of eliminating tissue debris and bacteria

Sodium hypochlorite ( $\mathrm{NaOCl}, 0.5-5.25 \%$ ) is a wellestablished irrigant for cleaning root canals because of both its antimicrobial activity and organic tissue dissolution capability. ${ }^{1,2}$ High concentrations ( $5 \%$ and higher) faster
DOI https://doi.org/ 10.1055/s-0040-1715985 ISSN $1305-7456$. (c) 2020. European Journal of Dentistry.

This is an open access article published by Thieme under the terms of the Creative Commons Attribution-NonDerivative-NonCommercial-License, permitting copying and reproduction so long as the original work is given appropriate credit. Contents may not be used for commercial purposes, or adapted, remixed, transformed or built upon. (https://creativecommons.org/licenses/by-nc-nd/4.0/)

Thieme Medical and Scientific Publishers Pvt. Ltd., A-12, 2nd Floor, Sector 2, Noida-201301 UP, India 
dissolve organic tissue but there are also concerns about their toxicity when inadvertently injected into periapical tissue. $^{3}$ Other studies showed that less concentrated solutions (1-3\%) maintain excellent antibacterial properties and that larger volumes and longer duration of irrigation have more impact on canal debridement. ${ }^{2}$ Thus, concentrations ranging between 2.5 and $3 \%$ are claimed to offer a good balance between efficiency and toxicity. Unfortunately, sodium hypochlorite has no effect on the inorganic fraction of dentin extracted during shaping, which contributes to the formation of the endodontic smear layer. ${ }^{4}$ This 0.5 to $2 \mu \mathrm{m}$-thick layer of organic and inorganic debris, packed onto canal walls, was shown to harbor bacteria and occlude $6 \%$ of the volume of mesial roots in mandibular molars., ${ }^{5,6}$ Thus, it is advocated to remove the smear layer in order to enhance canal disinfection..$^{7-10}$

Current clinical procedures rely on the use of sodium hypochlorite during shaping, followed by calcium complexing agents such as $17 \%$ ethylene diamine tetra-acetic acid (EDTA), to chelate calcium ions extracted from dentin., $4,11,12$ The efficiency of this combination of chemicals has long been demonstrated, but evidence also suggests that the cleanliness at the apical level of curved canals may be improved. ${ }^{13,14}$ Cutting debris accumulating along the inner curvature of root canals or occluding accessory canals are less likely to be removed or dissolved once they have formed..$^{15}$ One may argue that the chelating action of EDTA would be more useful during shaping to prevent the formation and accumulation of the smear layer onto canal walls, whereas the use of $\mathrm{NaOCl}$ would be more appropriate as a final disinfection step when shaping is completed.

In the current study, it is hypothesized that reversing the sequence of irrigants during shaping would lead to cleaner root dentin surfaces without altering its chemical composition. Specifically, scanning electron microscopy (SEM) was used to evaluate the cleanliness of root canals after different irrigation protocols, and energy-dispersive X-ray spectroscopy (EDX) was used to analyze chemical changes in dentin after treatment.

\section{Materials and Methods}

In accordance with the regulations of the Ethic Commission on Human Research of Geneva (CCER-Geneva), which authorizes the use of anonymous extracted teeth for in vitro research without written consent, 45 human necrotic single-rooted teeth were collected just after extraction. Teeth were radiographed from both buccolingual and proximal directions to determine canal curvature, according to the Schneider method. ${ }^{16}$ Only moderately curved canals $\left(10-30^{\circ}\right)$ were selected for the study and teeth exhibiting wide opened apices or straight roots were excluded. The Schneider method was used to measure canal curvature because it is widely in used in research and clinics to evaluate case difficulty. ${ }^{17}$

The access cavity was prepared using a cavity access $Z$ set (Dentsply Sirona Endodontics; Ballaigues, Switzerland), and root canal length was established using a \#10 K-file
(Micro-Mega; Besancon, France), pointing out the apical foramen, which were observed under the operatory microscope (Extaro 300 Carl Zeiss; Oberkochen, Germany); working length was defined $0.5 \mathrm{~mm}$ shorter. The apical foramen was sealed with cyanoacrylate glue (Zapit; Dental Ventures of America, Corona, California, USA) to reproduce a closed system. ${ }^{18}$ Canals were manually enlarged with \#15 K-file (Micro-Mega; Besancon, France) before using ProTaper Gold (Dentsply Sirona Endodontics; Baillaigues, Switzerland). Briefly, S1-S2-F1 and F2 progressively reached the working length, whereas the F3 was stopped $0.5 \mathrm{~mm}$ shorter. This final preparation $\left(0.3 \mathrm{~mm}\right.$ diameter, $9^{\circ}$ taper $)$ allowed the placement of the needle tip $3 \mathrm{~mm}$ shorter than the root canal terminus. ${ }^{19}$ The apical patency was verified between each rotary instrument using a \#10 K-file, which was allowed pass through the apical seal.

For all groups, a 30G open-ended needle (Miraject Endo Luer; Hager \& Werken, Duisburg, Germany) was used for canal irrigation; final solutions were manually activated (MDA: manual dynamic agitation) with a matching gutta-percha point (F3). Specimens were randomly divided into three groups $(n=15)$ that followed different irrigation protocols (see below).

For the standard irrigation group (SI), $0.5 \mathrm{~mL}$ of $3 \% \mathrm{NaOCl}$ was delivered between each instrument. This volume of irrigant was selected because a higher volume is unlikely to penetrate deep inside the root canal before shaping is completed. ${ }^{20} \mathrm{~A}$ final rinse of 1 minute was made using $5 \mathrm{~mL}$ of $17 \%$ EDTA, which was manually agitated for 30 seconds.

For the reverse irrigation group (RI), $0.5 \mathrm{~mL}$ of 17\% EDTA solution was used during shaping and renewed between each instrument. A final irrigation of 1 minute was made using $5 \mathrm{~mL}$ of $3 \% \mathrm{NaOCl}$ and manually agitated for 30 seconds.

Specimens of the reverse irrigation plus group (RI+) followed the same protocol as the reverse irrigation group, but the final irrigation using $5 \mathrm{~mL} \mathrm{NaOCl}$ was extended to 3 minutes and the solution manually agitated during the last 30 seconds. The rationale for using a prolonged application time was to increase the proteolytic action of sodium hypochlorite against canals debris that may contain a higher organic content.

All specimens were rinsed with $2 \mathrm{~mL}$ of distilled water to neutralize the final solution and stored at $4^{\circ} \mathrm{C}$.

\section{Preparation of the Samples for the Scanning Electron Microscopy (SEM)}

The specimens were embedded into polyvinylsiloxane material to produce individual molds designed to maintain the root during the splitting process necessary for SEM observation. A F3 gutta-percha point was fitted into the root canal to prevent the penetration of external debris. ${ }^{21}$ Longitudinal grooves (buccal and lingual) and transversal grooves were prepared to allow the placement of a scalpel blade (10; KLS Martin Group, Tuttlingen, Germany) that received a brief load in order to force the root to fracture. The samples were labeled, dehydrated (ascending concentrations of ethanol: $70 \%, 80 \%, 90 \%, 100 \%$, glued onto aluminum stubs, and gold sputtered. 


\section{Field Emission Scanning Electron Microscope (FESEM) Evaluation}

The samples were observed under a field emission scanning electron microscope (Zeiss Sigma 300 VP-FESEM; Oberkochen, Germany). A low-magnification (x50) was used to delineate two regions of interest, extending from 0 to $5 \mathrm{~mm}$ from the apex (apical) and from 5 to $10 \mathrm{~mm}$ (middle). The coronal portion of the root was excluded from evaluation because, independently of the irrigation protocol used, this region is easily cleaned..$^{14,22}$ For both regions, two microphotographs were taken (x350 and x1000) and evaluated by two calibrated observers, who blindly assessed the cleanliness of the canal using the Hulsmann score. ${ }^{13}$ In case of disagreement, the worst score was selected for calculations.

\section{EDX}

An Oxford X max 50 EDX spectroscopy system with dual silicon drift detectors, each with an area of $50 \mathrm{~mm}^{2}$, and a resolution of $125 \mathrm{eV}$ (Zeiss Sigma 300 VP-FESEM, Oberkochen, Germany) were used to measure the relative amounts of calcium, phosphorus, carbon, oxygen, sodium, and magnesium on dentin surfaces after treatment. EDX analysis was performed on each sample at the middle and apical regions. A mean value of the atomic percentage of each of the six elements that were measured across the root canal was calculated for the middle and apical regions.

\section{Statistical Analysis}

Mean scores and standard deviations were calculated for the apical and middle regions of each group and statistically compared using Kruskal-Wallis ANOVA by ranks and Wilcoxon matched pairs posthoc tests. A Chi-square test was used to assess whether the best cleanliness (score 1) would demonstrate a significant association with one of the three irrigation groups; odds ratio calculation was performed using score "1" versus score "2 or more" $(2+)$.

\section{Results}

Results indicate that the cleanliness of the middle and the apical regions were similar $(p>0.05)$, except for the RI group. As shown in - Fig. 1, mean scores observed in the middle region of the SI group $(1.87 \pm 0.74)$ and $\mathrm{RI}+$ group $(1.47 \pm 0.74)$ were statistically equivalent $(p>0.05)$, whereas the mean score for the RI+ group was significantly better $(p=0.014)$ than the RI group $(2.67 \pm 1.29)$. In the apical region, mean scores of the SI group (2.20 \pm 1.01$)$ and RI+ group (1.60 \pm 0.74$)$ were statistically equivalent but both significantly better than the mean score reported for the RI group $(3.53 \pm 1.19)$. Results of the odd ratios test indicate that the occurrence of score 1 at the apical region of RI+ group was significantly higher than RI and SI groups (respectively $p<0.000$ and 0.003 ).

SEM evaluation of the middle and the apical regions showed small amounts of smear layer, partially occluding dentinal tubules in SI group (-Fig. 2A and $\mathbf{2 B}$ ), whereas an amorphous surface layer was observed covering the root dentin of RI samples ( - Fig. 2C and 2D). RI+ samples showed no smear layer and opened dentinal tubules in both regions (-Fig. 2E and 2F). Independently of the irrigation protocol used, results from the EDX analysis showed that the relative percentages of the six elements measured on the middle and apical surfaces of the canal walls remained comparable. -Fig. 3A, 3B, 3C specifically shows the proportions of mineral (43.1-45.3\%) and organic elements (54.7-56.9\%) measured in the apical region after irrigation.

\section{Discussion}

The use of $\mathrm{NaOCl}$ followed by EDTA during biomechanical canal preparation is the gold standard.,11 This sequence of irrigation was used as a control in the SI group. Although this protocol was shown to allow clinicians to obtain clean canal surfaces in most instances, other reports suggest that

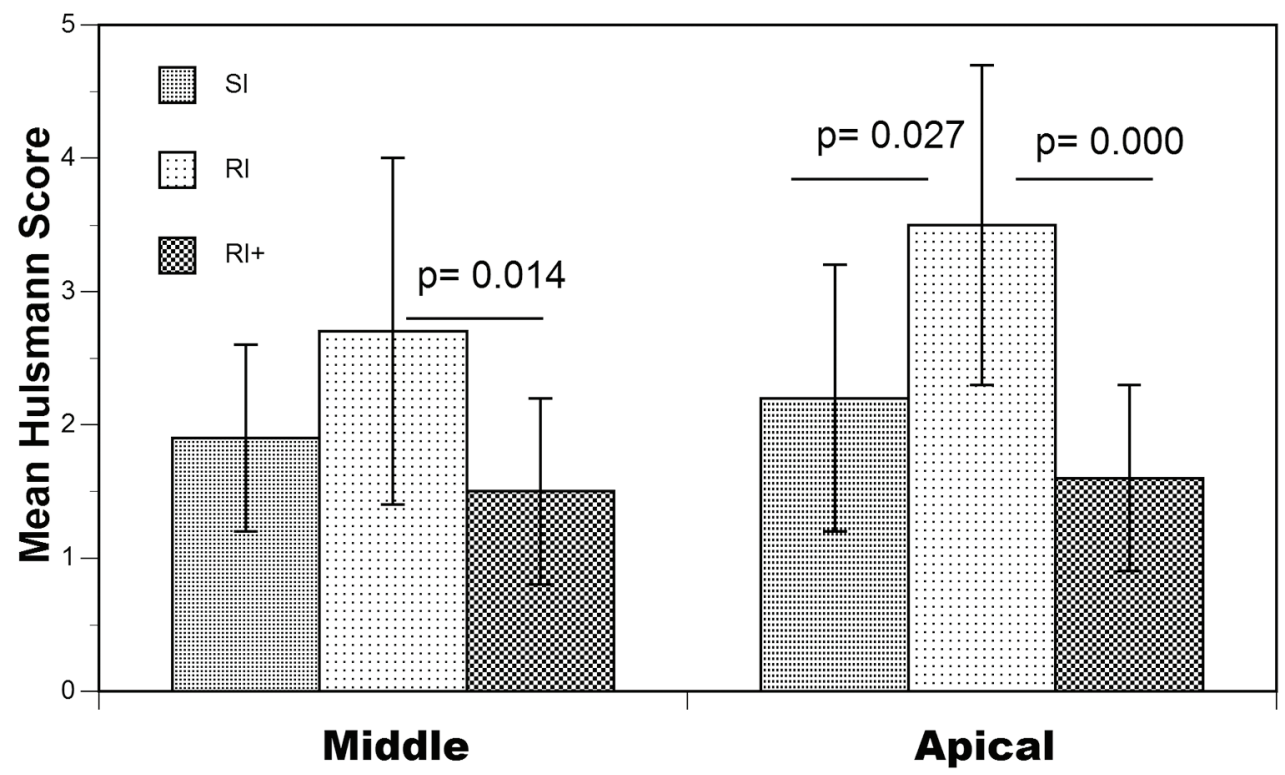

Fig. 1 Mean Hulsmann scores ( \pm standard deviation [SD]) in the middle and apical regions after standard irrigation (SI), reverse irrigation (RI) and reverse irrigation plus $(\mathrm{RI}+)$. 

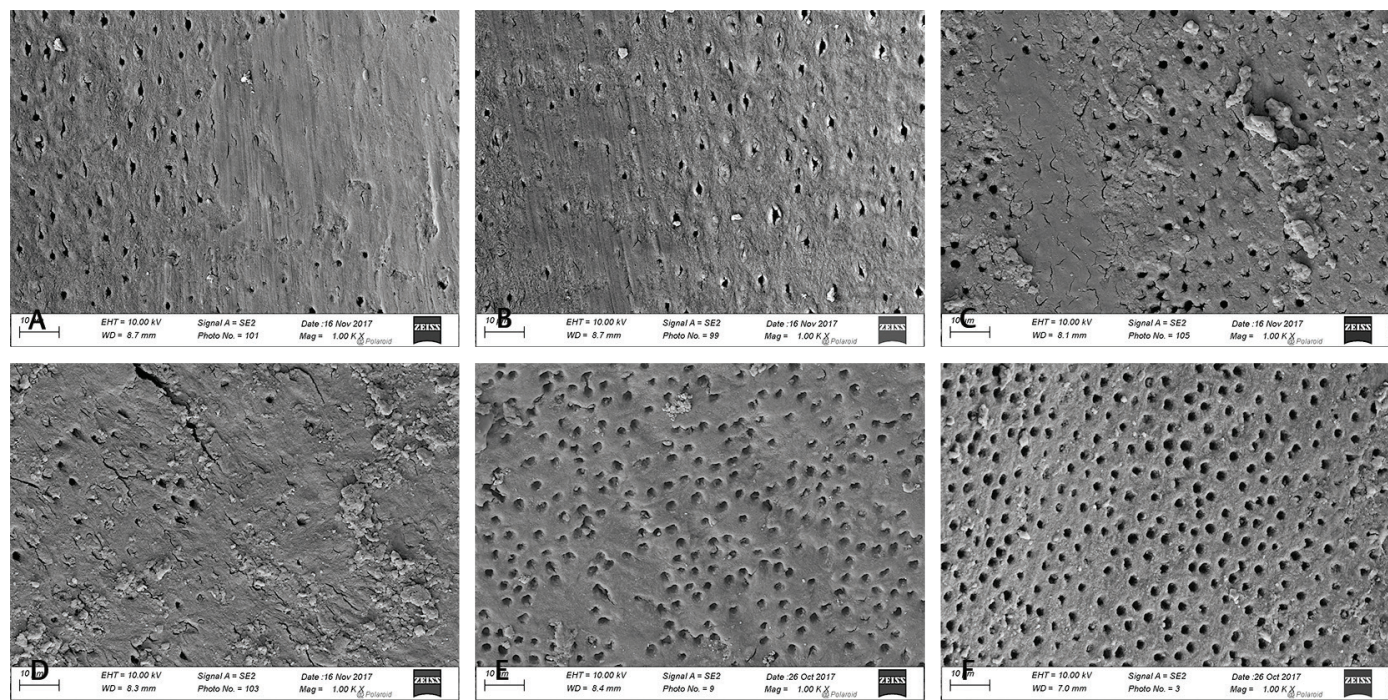

Fig. 2 SEM micrographs of: (A) Standard irrigation (SI) group, middle region x1000. (B) SI group, apical region x1000: the orifices of the dentin tubuli appear through a thin smear layer. (C) Reverse irrigation (RI) group, middle region $\times 1000$. (D) RI group, apical region $\times 1000$ : Large areas covered by an amorphous layer are visible. (E) RI+ group, middle region x1000. (F) RI+ group, apical region x1000: opened dentin tubuli and the absence of smear layer are observed.
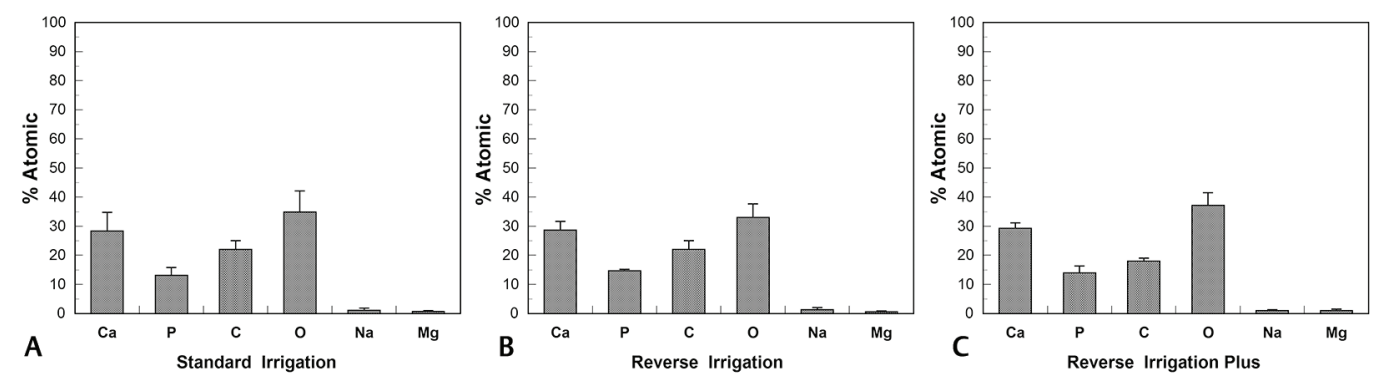

Fig. 3 Atomic percentage of calcium, phosphorous, carbon, oxygen, sodium, magnesium measured at: (A) apical region SI specimens, (B) apical region reverse irrigation (RI) specimens, and (C) apical region RI+ specimens.

cleaning the apical third of curved canals remains critical and advocate the use of supplementary procedures based on mechanic, sonic, ultrasonic, or laser devices. However, a recent meta-analysis that compared manual dynamic activation (MDA), passive ultrasonic irrigation (PUI), sonic irrigation (SI) or apical negative pressure (ANP) failed to identify any superiority among these different activation techniques. ${ }^{23}$ In the current study, we suggest reversing the sequence of irrigation to improve canal cleanliness without using supplemental instruments for activating irrigants.

Teeth used in this study had an intact or restored crown to provide a four-wall reservoir, promoting fluid exchange and renewal throughout shaping and irrigation. Selected root canals were moderately curved canals $\left(17^{\circ} \pm 6\right)$ because they are harder to clean apically. ${ }^{14,24}$ No statistical difference in canal length or canal curvature was found among the three experimental groups. The apical permeability was kept minimal to prevent the risk of excessive fluid communication between the root canal and the external environment that was shown to modify fluid dynamics during irrigation..$^{18}$ The use of the Protaper Gold system, comprising five successive instruments (S1 to F3), allowed the placement of a total volume of $2.5 \mathrm{~mL}$ of solution within five successive events. Boutsioukis et al reported that the $9 \%$ tapered apical preparation of Protaper F3 allows the deep placement of the irrigation needle and a higher shear stress to develop along canal walls during irrigation. ${ }^{25-27}$ Finally, a F3 gutta-percha point was used to manually agitate the solutions (MDA), in order to help the dispersion and mixing of irrigants in the stagnation zone and ensure that air inside the apical third (vapor-lock) was displaced..$^{28}$

Results for the SI group are in agreement with previously published reports that used the same scoring method. Whereas Caron et al reported a mean apical score of 2.21 in curved-canals, Ali-Ali et al obtained similar results after evaluating smear layer and debris removal effectiveness of several irrigation methods by combining the use of SEM and histological cross-sections. ${ }^{21,29}$ Recently, several authors have raised concerns about the use of qualitative scores to evaluate root canal cleanliness, mostly because the selection of the observed area is operator-dependent. It must be pointed out that in the current study, both examiners were blinded before scoring specimens and interexaminer reproducibility was verified using Kappa statistics $(K=0.82)$. More recently, the observation of root canals using micro-CT has been proposed to increase the quality of the results because the assessment of the same specimen before and after the experimental procedure is made possible. 
However, scanning and reconstruction procedures take a considerable amount of time and a perfect knowledge of dedicated software to provide accurate results. As reported by De Deus et al, the ideal experimental model to assess smear layer removal is not currently available. ${ }^{30}$

Interestingly, apical specimen exhibiting score 1 was 5.5 times more likely to be associated with RI+ than SI (OR 5\% 95\% confidence interval 3.12-9.68), supporting the superiority of this cleaning protocol. On the contrary, the RI tended to promote the accumulation of debris on canal walls ( - Fig. 2C and 2D) when compared with RI+ canal surfaces (-Fig. 2E and 2F). It is assumed that insufficient amounts of chlorine have been released to dissolve this organic rich layer. When the solution was left in place for 3 minutes (RI+) without increasing the total volume applied before activation for 30 seconds, this organic layer was no longer observed. ${ }^{31}$ In agreement with Zehnder et al in 2005, the prolonged use of sodium hypochlorite helps to wash out any residual EDTA before developing full proteolytic and antimicrobial activity. ${ }^{8}$

Although erosion of dentin consecutive to the use of EDTA followed by $\mathrm{NaOCl}$ has been reported by others, this phenomenon was not observed in the samples of the RI+ group. This could be explained by differences in EDTA/ $\mathrm{NaOCl}$ concentration ratios, $\mathrm{pH}$ and volume of solutions, duration of the reaction, and geometry of the specimens. ${ }^{32-34}$ As reported by Gu et al in 2017, dentin erosion mostly occurs when EDTA is applied onto NaOCl-treated dentin, as EDTA readily dissolves the collagen-depleted apatite crystallites at the surface of root dentin before propagating downward across the subsurface. Their results clearly indicated that high concentrations of hypochlorite ( $4 \%$ or higher) or extended irrigation times (30 minutes and more) are necessary to induce erosion of dentin. ${ }^{35}$ Also, in agreement with Calt and Serper, the application of EDTA either during (RI and RI+ groups) or after shaping (SI group), never exceeded 1 minute. ${ }^{36}$ Obviously, the risk that alterations in the chemical or structural composition of root dentin may have occur after a 3-minute period of application of $3 \% \mathrm{NaOCl}$ solution is unlikely. A lack of erosion is further supported by EDX analysis showing comparable mineral-organic ratios among groups and percentages of elements similar to others. ${ }^{33}$ Thus, a potential decline in the mechanical properties of $\mathrm{NaOCl}$-treated dentin can be ruled out when using 3\% sodium hypochlorite over 3 minutes. From a clinical point of view, it should be noted that the total volumes of irrigants applied in each group were strictly identical and that reversing the irrigation sequence would undoubtedly benefit the treatment of necrotic teeth where the tissue dissolving capability of $\mathrm{NaOCl}$ is less important compared with the antimicrobial activity.

Irrigation plays several roles during endodontic treatment, the most important being the elimination of endodontic pathogens causing infection; another role being the cleaning of the dentin surfaces covered by smear layer. As reported by Haapasalo et al in 2012, the smear layer has to be removed because it contains bacteria and bacterial antigens that were embedded into it during instrumentation of necrotic root canals. ${ }^{37}$ The smear layer also occludes dentin tubules, which are potentially infected and restrict the penetration of disinfecting solutions into dentin tubules. Wang et al in 2013 examined the effect of the smear layer on the antibacterial effect of different disinfecting solutions in infected dentinal tubules. ${ }^{38}$ They applied confocal laser scanning microscopy to detect live versus dead bacteria in the dentinal tubules after irrigation and concluded that the smear layer clearly decreases the antibacterial activity of $\mathrm{NaOCl}$ in dentin. More recently, Morago et al in 2016confirmed that that the presence of the smear layer significantly reduces the antimicrobial activity of $2.5 \% \mathrm{NaOCl} .{ }^{39}$ Thus, the cleanliness of the root canal walls and the quality of the final disinfection are strongly linked. Finally, there is evidence that a complete removal of the smear layer increases the sealing ability of several endodontic sealers. ${ }^{40}$

\section{Conclusions}

Using 17\% EDTA during shaping followed by $3 \% \mathrm{NaOCl}$ rinse for 3 minutes moderately improved apical cleanliness without inducing any sign of erosion on dentin canal walls. This irrigation protocol was significantly more likely to be associated with cleaner dentin than the SI protocol in the apical region. Further studies are needed to confirm this cleaning effect in teeth, exhibiting more complex anatomies such as transverse anastomosis, fins isthmus and lateral canals.

\section{Conflict of Interest}

None declared.

\section{References}

1 Sen BH, Piskin B, Demirci T. Observation of bacteria and fungi in infected root canals and dentinal tubules by SEM. Endod Dent Traumatol 1995;11(1):6-9

2 Siqueira JF, Jr, Rôças IN, Favieri A, Lima KC. Chemomechanical reduction of the bacterial population in the root canal after instrumentation and irrigation with $1 \%, 2.5 \%$, and $5.25 \%$ sodium hypochlorite. J Endod 2000;26(6):331-334

3 Cullen JK, Wealleans JA, Kirkpatrick TC, Yaccino JM. The effect of $8.25 \%$ sodium hypochlorite on dental pulp dissolution and dentin flexural strength and modulus. J Endod 2015;41(6):920-924

4 Yamada RS, Armas A, Goldman M, Lin PS. A scanning electron microscopic comparison of a high volume final flush with several irrigating solutions: Part 3. J Endod 1983;9(4):137-142

5 Paqué $\mathrm{F}$, Boessler C, Zehnder M. Accumulated hard tissue debris levels in mesial roots of mandibular molars after sequential irrigation steps. Int Endod J 2011;44(2):148-153

6 Violich DR, Chandler NP. The smear layer in endodontics - a review. Int Endod J 2010;43(1):2-15

7 Von der Fehr F, Nygaard Ostby B. Effect of EDTAC and sulfuric acid on root canal dentine. Oral Surg Oral Med Oral Pathol 1963;16:199-205

8 Zehnder M, Schmidlin P, Sener B, Waltimo T. Chelation in root canal therapy reconsidered. J Endod 2005;31(11):817-820

9 Holleman AF, Wiberg E, Inorganic Chemistry. San Diego: Academic Press; Berlin: W De Gruyter. 2001

10 Byström A, Sundqvist G. The antibacterial action of sodium hypochlorite and EDTA in 60 cases of endodontic therapy. Int Endod J 1985;18(1):35-40

11 Zehnder M. Root canal irrigants. J Endod 2006;32(5):389-398

12 Hülsmann $M$, Heckendorff $M$, Lennon $A$. Chelating agents in root canal treatment: mode of action and indications for their use. Int Endod J 2003;36(12):810-830 
13 Hülsmann M, Rümmelin C, Schäfers F. Root canal cleanliness after preparation with different endodontic handpieces and hand instruments: A comparative SEM investigation. J Endod 1997;23(5):301-306

14 Gambarini G, Laszkiewicz J. A scanning electron microscopic study of debris and smear layer remaining following use of GT rotary instruments. Int Endod J 2002;35(5):422-427

15 Haupt F, Meinel M, Gunawardana A, Hülsmann M. Effectiveness of different activated irrigation techniques on debris and smear layer removal from curved root canals: A SEM evaluation. Aust Endod J 2020;46(1):40-46

16 Schneider SW. A comparison of canal preparations in straight and curved root canals. Oral Surg Oral Med Oral Pathol 1971;32(2):271-275

17 Faraj S, Boutsioukis C. Observer variation in the assessment of root canal curvature. Int Endod J 2017;50(2):167-176

18 Parente JM, Loushine RJ, Susin L, et al. Root canal debridement using manual dynamic agitation or the EndoVac for final irrigation in a closed system and an open system. Int Endod J 2010;43(11):1001-1012

19 Boutsioukis C, Van der Sluis LWM, Syringe irrigation: blending endodontics and fluid dynamics. In: Endodontic Irrigation, Chemical Disinfection of the Root Canal System Springer International Publishing; 2015 45-6

20 Bronnec F, Bouillaguet S, Machtou P. Ex vivo assessment of irrigant penetration and renewal during the cleaning and shaping of root canals: a digital subtraction radiographic study. Int Endod J 2010;43(4):275-282

21 Caron G, Nham K, Bronnec F, Machtou P. Effectiveness of different final irrigant activation protocols on smear layer removal in curved canals. J Endod 2010;36(8):1361-1366

22 Saito K, Webb TD, Imamura GM, Goodell GG. Effect of shortened irrigation times with $17 \%$ ethylene diamine tetra-acetic acid on smear layer removal after rotary canal instrumentation. J Endod 2008;34(8):1011-1014

23 Virdee SS, Seymour DW, Farnell D, Bhamra G, Bhakta S. Efficacy of irrigant activation techniques in removing intracanal smear layer and debris from mature permanent teeth: a systematic review and meta-analysis. Int Endod J 2018;51(6):605-621

24 Senia ES, Marshall FJ, Rosen S. The solvent action of sodium hypochlorite on pulp tissue of extracted teeth. Oral Surg Oral Med Oral Pathol 1971;31(1):96-103

25 Khademi A, Yazdizadeh M, Feizianfard M. Determination of the minimum instrumentation size for penetration of irrigants to the apical third of root canal systems. J Endod 2006;32(5):417-420

26 Sedgley CM, Nagel AC, Hall D, Applegate B. Influence of irrigant needle depth in removing bioluminescent bacteria inoculated into instrumented root canals using real-time imaging in vitro. Int Endod J 2005;38(2):97-104

27 Boutsioukis C, Gogos C, Verhaagen B. Versluis M, Kastrinakis E, Van der Sluis LW. The effect of root canal taper on the irrigant flow: Evaluation using an unsteady Computational Fluid Dynamics model. Int Endod J 2010;43(10):909-916

28 Saber Sel-D, Hashem AA. Efficacy of different final irrigation activation techniques on smear layer removal. J Endod 2011;37(9):1272-1275

29 Al-Ali M, Sathorn C, Parashos P. Root canal debridement efficacy of different final irrigation protocols. Int Endod J 2012;45(10):898-906

30 De-Deus G, Reis C, Paciornik S. Critical appraisal of published smear layer-removal studies: methodological issues. Oral Surg Oral Med Oral Pathol Oral Radiol Endod 2011;112(4):531-543

31 Rossi-Fedele G, Doğramaci EJ, Guastalli AR, Steier L, de Figueiredo JA. Antagonistic interactions between sodium hypochlorite, chlorhexidine, EDTA, and citric acid. J Endod 2012;38(4):426-431

32 Qian W, Shen Y, Haapasalo M. Quantitative analysis of the effect of irrigant solution sequences on dentin erosion. J Endod 2011;37(10):1437-1441

33 Wang Z, Maezono H, Shen Y, Haapasalo M. Evaluation of root canal dentin erosion after different irrigation methods using energy dispersive X-ray spectroscopy. J Endod 2016;42(12):1834-1839

34 Niu W, Yoshioka T, Kobayashi C, Suda H. A scanning electron microscopic study of dentinal erosion by final irrigation with EDTA and $\mathrm{NaOCl}$ solutions. Int Endod J 2002;35(11):934-939

$35 \mathrm{Gu}$ LS, Huang XQ, Griffin B, et al. Primum non nocere - The effects of sodium hypochlorite on dentin as used in endodontics. Acta Biomater 2017;61:144-156

36 Calt S, Serper A. Time-dependent effects of EDTA on dentin structures. J Endod 2002;28(1):17-19

37 Haapasalo M, Qian W, Shen Y. Irrigation: beyond the smear layer. Endod Topics 2012;27:35-53

38 Wang Z, Shen Y, Haapasalo M. Effect of smear layer against disinfection protocols on Enterococcus faecalis-infected dentin. J Endod 2013;39(11):1395-1400

39 Morago A, Ordinola-Zapata R, Ferrer-Luque CM, Baca P, Ruiz-Linares M, Arias-Moliz MT. Influence of smear layer on the antimicrobial activity of a sodium hypochlorite/etidronic acid irrigating solution in infected dentin. J Endod 2016;42(11):1647-1650

40 Clark-Holke D, Drake D, Walton R, Rivera E, Guthmiller JM. Bacterial penetration through canals of endodontically treated teeth in the presence or absence of the smear layer. J Dent 2003;31(4):275-281 